\title{
EDITORIAL
}

\section{Quantum description of transport phenomena: Recent progress}

\author{
Wei Ji (季威 $)^{1, *}$, Hong-Qi Xu (徐洪起 $)^{2,3, \dagger}$, Hong Guo (郭鸿 $)^{4, \#}$ \\ ${ }^{1}$ Department of Physics and Beijing Key Laboratory of Optoelectronic Functional Materials 83 Micro-Nano Devices, \\ Renmin University of China, Beijing 100872, China \\ ${ }^{2}$ Key Laboratory for the Physics and Chemistry of Nanodevices and Department of Electronics, Peking University, \\ Beijing 100871, China \\ ${ }^{3}$ Division of Solid State Physics, Lund University, Box 118, S-22100 Lund, Sweden \\ ${ }^{4}$ Centre for the Physics of Materials and Department of Physics, McGill University, Montreal, QC, Canada H3A $2 T 8$ \\ E-mail: *wji@ruc.edu.cn, †hqxu@pku.edu.cn, \#guo@physics.mcgill.ca \\ Received November 27, 2014
}

Over 100 years ago, atoms were believed to be the smallest indivisible particles and no one knew what was inside an electric current. For instance, what was cathode ray made of had puzzled many. In 1897, J. J. Thomson, based on his refined and newly designed experiments, discovered that cathode rays were made of electrons! Fifty years later, William Shockley, John Bardeen, and Walter Brattain invented a device called transistor that operated on the flow of electrons. A revolution of information technology started.

Exactly 67 years after the invention of transistor, here we are at the east campus of Renmin University of China in Beijing, enjoying a beautiful November afternoon. Almost every student walking by is busy tapping some hand held device. In a modern city like Beijing, probably, every person has a computer, a pad, a smartphone or some electronic gadget each containing billions of transistors. The materials of building transistors have evolved from a piece of germanium, plastic and gold in 1947 to today's silicon, high-k oxide and copper, and will inevitably move forward to a "post-silicon" era with emerging electronic materials. The information carrier, originally electric charge, now includes spin, possibly "valley", and perhaps other more exotic quantum degrees of freedom by the on-going effort of physicists. Profiting from nanofabrication, the characteristic size of a transistor has decreased from the millimeter scale to micrometer scale and now to nanometer scale, closely following the Moore's Law. The perspective of eventually reaching the single atom or molecule scale is exciting. Semiconductor field effect transistor (FET) is the most widely used transistor technology today. In an FET, electrons flow through the semiconductor channel in a controlled manner. For long channels, electron motion is interrupted by frequent random collisions with such things as impurities, defects and vibrations of the material lattice, and transport can be well described by diffusion. On the other hand, for very short channels, electrons sip through without enough time to suffer any collision, and transport is ballistic. To move the device technology forward, achieving a basic understanding of carrier transport is of critical importance.

There has been a long history of theoretical description of transport processes. Boltzmann was the first one thinking of combining Newtonian mechanics with entropy driven processes to analyze transport phenomenon. The Boltzmann transport equation which combines classical mechanics with statistical mechanics is the standard semi-classical theory for predicting transport properties of electronic devices. Similarly, a full quantum transport theory requires some kind of combination of quantum mechanics with statistical mechanics, and is conveniently done by the non-equilibrium Green's function (NEGF) formalism established by the pioneering work of Martin, Schwinger, Kadanoff, Baym, Keldysh, and the others. More recently, Landauer, Buttiker, and Datta contributed significantly on our current understanding of quantum transport at the nanoscale [1]. Taylor, Guo, and Wang were the first to establish first principles materials calculation by density functional theory (DFT) within the framework of NEGF [2] and produced the first NEGF-DFT quantum transport package named McDCal [2] - abbreviation of McGill Devices Calculator, offering full quantum description of transport phenomena for real materials.

After 14 years of the first report of NEGF-DFT formalism and code, this approach has become the de facto method for parameter-free simulations of quantum transport and device physics. Many implementations of NEGF-DFT, including MatDCal, NanoDCal, Transiesta, ATK, SMEAGOL and several others, are now widely used by physicists, chemists, materials sci- 
entists and electronics scientists/engineers. From 2011 to 2014, we have organized four workshops for emerging electronic materials and devices (EEMD) in China, discussing recent progress of quantum transport modeling with researchers from diverse backgrounds [3]. These workshops reveal that many research groups are pushing the boundaries of the NEGF-DFT formalism to solve increasingly wider range of quantum transport problems in many different fields. These and other achievements in quantum transport modeling deserve to be documented in a dedicated collection.

This special issue of "Recent Progress of Quantum Transport Theory and its Applications" reports recent progress of quantum modeling in terms of both methodological development and applications. Reviews and original research papers included here cover a broad range of topics:

- Methodological aspects. The NEGF method applied to the problems of energy transport due to atomic vibrations (phonons) is reviewed in Ref. [4] which is highly relevant for thermoelectricity. The theoretical foundation and numerical algorithm for hierarchical equation of the motion of reduced single electron density matrix is summarized in Ref. [5] to address time-dependent quantum transport phenomenon. A three-dimensional (3D) atom-superposition approach for simulating scanning tunneling microscopy (STM) and spectroscopy (STS) is presented in Ref. [6]. This 3D Wentzel-Kramers-Brillouin (WKB) model is particularly suitable for simulating spin-polarized STM and STS which are advanced experimental techniques for surface magnetism.

- Molecular systems. In a mini review [7], all carbonbased molecular junctions were discussed including mechanically controlled conductance switches, negative differential resistances and electronic rectifiers. A research article [8] addresses a longstanding issue for molecular transport junction, namely whether the $\mathrm{H}$ atom is dissociative from the SH group in an Au-Benzenedithiol-Au structure. This article reports some compelling evidence that $\mathrm{H}$ remains. Interestingly, a theoretical study [9] indicates that DNA, a very large molecule, may well have huge potential for applications of quantum interference.

- Roles for spin. Spin transport at the nanoscale is now termed spintronics. Both research articles [10, 11] discuss aspects of quantum transport of spins. In particular, helical states of spins are demonstrated in a topological insulator $\mathrm{Bi}_{2} \mathrm{Se}_{3}$ [10], while spin transfer torque is discussed in a prototypical magnetic tunnel junction [11].

This Special Issue is intended to provide physics community with a multi-disciplinary understanding of methodological development and applications of NEGFDFT, and to bring about new ideas for expanding the applicability of quantum transport theory and numerical methods. The ultimate goal is, of course, to deepen our understanding of quantum transport phenomena in the nano-scale and to help the advancement of device physics.

We thank all contributors and referees whose hard work and devotion made this Special Issue possible.

\section{References and notes}

1. Supriyo Datta, Lessons from Nanoelectronics: A New Perspective on Transport, Singapore: World Scientific Publishing Company, 2012, and the references therein.

2. J. Taylor, H. Guo, and J. Wang, Ab initio modeling of quantum transport properties of molecular electronic devices, Phys. Rev. B, 2001, 63(24): 245407

3. For more details, please visit: http://www.eemd.cn/

4. J.-S. Wang, B. Kumar Agarwalla, H. Li, and J. Thingna, Nonequilibrium Green's function method for quantum thermal transport, Front. Phys., 2014, 9(6): 673

5. Y. Kwok, Y. Zhang, and G.-H. Chen, Time-dependent density functional theory for quantum transport, Front. Phys., 2014, 9(6): 698

6. K. Palotás, G. Mándi, and W. A. Hofer, Three-dimensional Wentzel-Kramers-Brillouin approach for the simulation of scanning tunneling microscopy and spectroscopy, Front. Phys., 2014, 9(6): 711

7. X.-F. Li and Y. Luo, Conductivity of carbon-based molecular junctions from ab-initio methods, Front. Phys., 2014, 9(6): 748

8. Z.-Y. Ning, J.-S. Qiao, W. Ji, and H. Guo, Correlation of interfacial bonding mechanism and equilibrium conductance of molecular junctions, Front. Phys., 2014, 9(6): 780

9. W. Zhu, A.-M. Guo, and Q.-F. Sun, Electronic transport through tetrahedron-structured DNA-like system, Front. Phys., 2014, 9(6): 774

10. Y.-B. Hu, Y.-H. Zhao, and X.-F. Wang, A computational investigation of topological insulator $\mathrm{Bi}_{2} \mathrm{Se}_{3}$ film, Front. Phys., 2014, 9(6): 760

11. X.-T. Jia and K. Xia, Electric and thermo spin transfer torques in $\mathrm{Fe} /$ Vacuum/Fe tunnel junction, Front. Phys., 2014, 9(6): 768 